\title{
Evaluación de tratamientos superficiales en madera expuesta a condiciones de intemperismo natural
}

\author{
Taraborelli, Carla ${ }^{1,4}$; M. Mercedes Refort ${ }^{1}$; Eleana Spavento ${ }^{1}$; Laura Maly ${ }^{1}$; Luis Acuña ${ }^{2}$; \\ Ricardo Camera ${ }^{3}$; Gabriel Keil ${ }^{1}$ \\ ${ }^{1}$ Laboratorio de Investigaciones en Maderas (LIMAD). Facultad de Ciencias Agrarias y Forestales (FCAyF). \\ Universidad Nacional de la Plata (UNLP). Argentina; ${ }^{2}$ Laboratorio de Maderas. Universidad de Valladolid, \\ Palencia, España; ${ }^{3}$ Profesional Independiente, Química Bosques S.A., Florencio Varela, Buenos Aires, \\ Argentina; ${ }^{4}$ carla.taraborelli@agro.unlp.edu.ar
}

Taraborelli, Carla; M. Mercedes Refort; Eleana Spavento; Laura Maly; Luis Acuña; Ricardo Camera; Gabriel Keil (2020) Evaluación de tratamientos superficiales en madera expuesta a condiciones de intemperismo natural. Rev. Fac. Agron. Vol 119 (1): 1-9. https://doi.org/10.24215/16699513e039

\begin{abstract}
La madera al exterior sufre intemperismo, proceso superficial causado principalmente por radiación solar (UV), humedad relativa, temperatura y precipitaciones, resultando la aparición de micro-macrogrietas, aspecto rugoso y agrisado superficial. El objetivo fue analizar cualitativamente el comportamiento de 11 recubrientes, aplicados sobre madera de Pinus ponderosa expuesta al exterior en la ciudad de La Plata, Argentina. Se ensayaron 9 productos disponibles en el mercado: 2 esmaltes sintéticos, 3 barnices, 3 lasures yun esmalte acrílico,dos productos en desarrollo y un testigo sin recubrir. Se realizaron evaluaciones visuales y registros fotográficos cada 30 días durante 21 meses, en base a una grilla de 6 variables: cambio de brillo y de color, agrietamientos, manchado de clavos, presencia de mohos y desgaste del recubriente, valoradas en escala de 0 a 4. Transcurridos los 21 meses de exposición, se observaron diferencias significativas entre los recubrientes, siendo los esmaltes sintéticos los de mejor comportamiento, arrojando valores entre 1,5 y 4, mientras que los barnices y el testigo arrojaron resultados entre 0,2 y 1,1 dependiendo de la variable, evidenciando una pérdida total de su vida útil. En un plano intermedio, los lasures comerciales y en desarrollo arrojaron valores entre 1,1 y 3,3 . Se concluye que no existe un tipo de recubriente que atienda a todos los factores abióticos y que no sufra la acción del intemperismo.
\end{abstract}

Palabras clave: envejecimiento, deterioro, vida útil, Pinus ponderosa

Taraborelli, Carla; M. Mercedes Refort; Eleana Spavento; Laura Maly; Luis Acuña; Ricardo Camera; Gabriel Keil (2020) Evaluation of surface coatings on wood exposed to natural weathering conditions Rev. Fac. Agron. Vol 119 (1): 1-9. https://doi.org/10.24215/16699513e039

\begin{abstract}
The wood exposed to solar radiation (UV), relative air humidity $(\mathrm{RH})$, temperature, precipitation, among other suffers a process called weathering; as a result appear cracks, surface roughness and graying color of wood. The aim of this study was to analyze qualitatively the behavior of 11 coatings on Pinus ponderosa (Dougl. ex Laws)wood exposed in La Plata, Argentina. There were tested: 9 market products -2 synthetic enamels; 3 varnishes; 3 lasures and acrylic enamel- two lasures products in development, and an uncoated witness, a total of 12 treatments. Visual evaluations and photographic records are carried out every 30 days for 21 months, based on 6 variables: change of brightness and color, cracks, iron tanatos of the nails, fungi and wear of the coating, valued on a scale from 0 to 4 . After 21 months of exposure, significant differences were observed between the coats. The synthetic glazes are the best performers, with values between 1.5 and 4 , while varnishes and control yielded results between 0.2 and 1.1 depending on the variable, evidencing a loss of its useful life. The lasures had an intermediate behavior yielded values between 1.1 and 3.3. It is concluded there is no coating that cover all abiotic requirements and that does not suffer the action of weathering.
\end{abstract}

Key words: ageing, deterioration, useful life, Pinus ponderosa.

https://revistas.unlp.edu.ar/revagro

Recibido: $18 / 02 / 2019$

Aceptado: 29/11/2019

Disponible on line: $01 / 07 / 2020$

ISSN 0041-8676 - ISSN (on line) 1669-9513, Facultad de Ciencias Agrarias y Forestales, UNLP, Argentina 


\section{INTRODUCCIÓN}

La madera expuesta al exterior sufre en la capa superficial un deterioro físico y químico lento, provocado por las condiciones ambientales, denominado intemperismo (Chang et al., 1982). Este proceso es causado por diversos agentes tales como radiación solar, humedad relativa, temperatura, precipitaciones (lluvia, nieve, granizo), abrasión provocada por las partículas arrastradas por el viento, actividades humanas, entre otros. El agente de mayor impacto superficial es la radiación solar, a través de la radiación ultravioleta (UV: 295-400 nm),que fotodegrada e induce cambios químicos en los componentes estructurales de la madera (lignina, celulosa y hemicelulosa), con especial énfasis en la lignina, y que a su vez, junto con los rayos infrarrojos, tienen la capacidad de degradar los extractivos presentes en la misma (Chang et al., 1982; Anderson et al., 1991; Flores-Velázquez et al., 2001; Evans et al., 2005; Williams, 2005). En términos generales la radiación UV causa disociación de enlaces de la lignina, generando hidroperóxidos, que se desdoblan en grupos carbonilos (Rodríguez-Anda \& Fuentes Talavera, 2003; Gascón-Garrido et al., 2016).

A nivel macroscópico el resultado del proceso se visualiza a través de micro y macrogrietas, aspecto rugoso de la superficie producto de la separación de las fibras de celulosa y un color agrisado característico del intemperismo. Asimismo, se observa un fenómeno de colonización por microorganismos, tales como hongos causantes de mancha azul y mohos, ya que la degradación de los componentes principales de la madera proporciona los nutrientes específicos que estos agentes fúngicos metabolizan para su crecimiento (Gascón-Garrido et al., 2016). Por su parte, a nivel microscópico ocurre una disminución en la cantidad de los principales componentes de la madera (celulosa, hemicelulosa, lignina yextractivos), visualizándose una separación de los elementos celulares por destrucción de la laminilla media, destrucción de punteaduras areoladas y grietas en las paredes celulares (Williams, 2005).

Si bien el fenómeno de intemperismo no proporciona directamente una disminución en las propiedades mecánicas de la madera, a partir de éste pueden devenir efectos secundarios. De esta forma los cambios dimensionales, producto de las variaciones de las condiciones higrotérmicas, pueden generar grietas y/o rajaduras que constituyan, conjuntamente con un mayor contenido de humedad de la madera, un ambiente propicio para la colonización de agentes fúngicos (xilófagos) que degraden el material, disminuyendo así dichas propiedades.

Con el fin de evitar y/o retardar los efectos generados por el intemperismo, existen productos que se aplican sobre la superficie de la madera, denominados recubrientes superficiales (Keil et al., 2016). En términos generales se pueden describir tres tipos de recubrientes utilizados en la madera: esmaltes, barnices y lasures. Los esmaltes son pinturas formadoras de películas (poro cerrado) que no permiten visualizar la veta de la madera. Están formados por pigmentos sólidos, aglutinantes y secantes (Garay Moena, 2007). Los barnices por su parte son productos compuestos por aglutinantes, plastificantes, disolventes y diluyentes que posterior a su aplicación y en estado seco, originan una película insoluble al agua, adherente, dura, generalmente lisa y brillante que permite visualizar la veta de la madera (Tamarit-Urias et al., 2003). Los lasures o stains son recubrimientos transparentes o semi-transparentes que no forman una película y presentan un acabado a poro abierto que resulta un regulador de la humedad de la madera (UNE-EN 927-1, 2000). Son productos en base orgánica, solubles o insolubles al agua que presentan pigmentos minerales que reflejan la radiación UV, responsable del agrietamiento y del color grisáceo de la madera (Garay Moena, 2007).

Si bien todos estos recubrientes superficiales le otorgan un aumento en la vida útil de la madera, ésta depende también del efecto que el intemperismo ejerce sobre ellos y de las propiedades intrínsecas del material utilizado (Gascón-Garrido et al., 2016). De este modo, características del material como porosidad, contenido de resinas, densidad, grano inclinado, diferencias entre leño temprano y tardío, nudos, pueden influir en la correcta incorporación y anclaje del recubrimiento en la madera, como así también en su efectividad y permanencia durante la vida útil; características dependientes y variables acorde con la especie maderera en que se apliquen (Flores-Velázquez et al., 2001; Taraborelli, 2016).

Al respecto, en la actualidad, son las especies forestales de bosques cultivados las que mayor representatividad tienen en los productos madereros comerciales. Dichas especies, en términos generales, presentan propiedades de durabilidad natural que limitan su vida útil en servicio, siendo para ello necesario, comoalternativa,el uso de estos productos que permitan aumentarle su vida útil en usos exteriores. Un ejemplo de ello lo constituye la madera de Pinus ponderosa Douglas ex. Laws procedente de la región Patagónica Argentina. Los principales productos de esta madera presentes en el mercado local tienen como destino la construcción, tanto en material cilíndrico, postes cabañeros, como en material aserrado: tablas, tirantes, moldurados comomachimbres y productos media caña simulando un poste cabañero para revestimientos exteriores; como así también, vigas laminadas y ladrillos de madera (Keil et al., 2015). Muchos de estos usos requieren de resistencia al deterioro por factores abióticos, por lo que resulta relevante conocer los productos para preservarlos, de acuerdo a la zona, exposición y propósito buscado, logrando así la mayor permanencia del producto en el material y el manteniendo de sus características originales en el tiempo.

De acuerdo con lo expuesto, el objetivo de este trabajo fue analizar cualitativamente el comportamiento de distintos productos de recubrimiento presentes en el mercado local y en desarrollo, aplicados sobre madera de pino ponderosa (Pinus ponderosa Douglas ex. Laws) expuesta al exterior en la ciudad de La Plata, Argentina. 


\section{MATERIALES Y MÉTODOS}

\section{Material leñoso}

El sustrato empleado fue madera comercial de pino ponderosa de 29 años de edad, proveniente de la Empresa CORFONE SA (Junín de los Andes, Neuquén), quien proveyó el material aserrado, seco y cepillado al Laboratorio de Investigaciones en Madera (LIMAD - FCAyF). Para los estudios se tomó como referencia la norma UNE-EN 927-3 (2000).

\section{Productos recubrientes}

Se realizaron 12 tratamientos, 11 de ellos con productos aportados por la empresa Química Bosques S.A.: 9 productos disponibles en el mercado, dos productos en desarrollo y un testigo. Se trabajó con 6 probetas por tratamiento.

Los productos de mercado ensayados fueron: 1) esmalte sintético al solvente,2) esmalte sintético al agua,3) barniz sintético para madera, 4) barniz marino doble filtro solar, 5) barniz revestimiento para madera,6) lasur protector para maderas, 7) lasur classic, 8) lasur protector para maderas exterior, 9) esmalte acrílico de máxima duración. Los dos productos en desarrollo ensayados fueron: 10) lasur semitransparente y 11) lasur alto sólido.

\section{Aplicación del producto}

La aplicación se llevó a cabo mediante pincelado, siguiendo las especificaciones detalladas en el producto, por lo que, para lograr el rendimiento indicado se empleó la concentración, tipo de diluyente, preparación de la superficie, cantidad de aplicaciones y horas de fraguado recomendados en cada uno de ellos.

\section{Instalación del ensayo}

El ensayo fue llevado a cabo en la Estación Experimental J. Hirschhörn (EEJH) de la FCAyF UNLP, La Plata, Argentina (3498'43"S, 57 99'64" W). Para la instalación del mismo se construyó una estructura de hierro con una inclinación de $45^{\circ} \mathrm{y}$ orientada al Norte geográfico. Para evitar la invasión de malezas en la base del ensayo se colocó un polietileno negro de 200 micrones, evitando la utilización de herbicidas que podrían influenciar en los resultados del ensayo. En la estructura se instaló un bastidor de madera dura y durable, Cabralea canjerana subsp. polytricha (A.Juss.) T.D.Penn., conteniendo cinco probetas por tratamiento unidas mediante clavos; la sexta probeta fue reservada en laboratorio y utilizada para realizar comparación visual de las variables durante el ensayo. Las condiciones medias de temperatura, precipitación total, radiación solar y humedad relativa en el período de evaluación fueron: $16^{\circ} \mathrm{C} ; 96,2 \mathrm{~mm} ; 4239,2 \mathrm{wat} / \mathrm{m}^{2}$ y $81 \%$, respectivamente.

\section{Evaluación de productos recubrientes- intemperismo}

La valoración se realizó de manera cualitativa mediante la comparación visual y registro fotográfico de cada probeta/tratamiento. Para la toma de datos se utilizó una grilla de 6 variables estableciendo una escala de 0 (pérdida total de vida útil) a 4 (sin pérdida de vida útil). Las variables relevadas fueron: brillo, color, agrietamiento de la probeta, manchas de tanato de hierro de los clavos, hongos y desgaste de recubriente. La toma de datos se realizó cada 30 días durante 21 meses.

\section{Análisis de resultados}

En cada una de las seis variables analizadas se realizó un modelo de efectos mixtos para describir el análisis de medidas repetidas. A fin de evaluar la relación entre las variables cualitativas, se realizó una correlación Ordinal de Spearman. Posteriormente se ajustó un modelo considerando aleatoriamente, las variables o parámetros visuales tratamiento y tiempo (mes). Se realizaron gráficos de "índice de vida útil" ponderando para ello dichos parámetros visuales considerados por producto. En cada caso se utilizó la función "jitter" para visualizar mejor los valores superpuestos. Se realizó un Test de Tukey para evaluar diferencias significativas entre las variables. Todos los análisis estadísticos fueron llevados a cabo con el Software $R^{\odot}(3.4 .4$ versión) (R Development CoreTeam, 2018).

\section{RESULTADOS}

En Tabla 1 se presentan los resultados del Test de Tukey para cada recubriente según la variable de estudio.

\section{Cambio de Color, brillo y desgaste}

La correlación de Spearman realizada para las tres variables arrojó p-valores inferiores a 0,05 y coeficiente de correlación positivo, por lo que puede indicarse que las variables están correlacionadas significativa y positivamente. En el Test de Tukey los resultados arrojados por estas variables son similares, por lo queen esta sección se presentará el gráfico de cambio de color, siendo el gráfico de desgaste y cambio de brillo análogos (no presentados).

Los resultados demostraron que el producto no comercial en desarrollo (10), el esmalte sintético al solvente (1) y el esmalte sintético al agua (2) se diferencian significativamente del resto de los recubrientes (Tabla 1). Asimismo, presentaron un comportamiento similar en las tres variables estudiadas (Figura 1) y se diferenciaron en los tres casos del resto de los recubrientes. En términos generales el material tratado con barniz y el testigo arrojaron valores muy bajos en todas las variables analizadas dando como resultado un comportamiento deficiente de los recubrientes, ya que en 6 meses de exposición habían perdido completamente su funcionalidad; contrariamente con esto, los acabados de esmalte sintético al solvente (1) y esmalte sintético al agua (2) no perdieron su vida útil en ese mismo período. En un punto intermedio se encontraron los lasures comerciales y no comerciales, los cuales no perdieron en totalidad el color y el brillo y, si bien manifestaron cierto desgaste, continuaron funcionales hasta finalizar el ensayo con 21 meses de exposición.

Los recubrientes de color blanco se comportaron mejor que aquellos con tonalidad, comportamiento posiblemente relacionado con la absorción y reflexión de energía dada por los rayos UV de dicho pigmento. 
Tabla 1. Resultados de los Test de Tukey correspondientes a cada variable. Referencias: *T=Tratamiento. 1) esmalte sintético al solvente, 2) esmalte sintético al agua, 3) barniz sintético para madera, 4) barniz marino doble filtro solar, 5) barniz revestimiento para madera, 6) lasur protector para maderas, 7) lasur classic, 8) lasur protector para maderas exterior, 9) esmalte acrílico de máxima duración, 10) lasur semitransparente, 11) lasur alto sólido y 12) Testigo. ${ }^{* *} p=p$ value. Los valores indican la escala de 0 a 4, siendo 0 pérdida de vida útil y 4 permanencia de vida útil, mientras que valores intermedios significan el gradiente de pérdida de vida útil a lo largo del tiempo. Letras iguales indican diferencia no significativa.

\begin{tabular}{|c|c|c|c|c|c|c|}
\hline$T^{*}$ & $\begin{array}{l}\text { Cambio } \\
\text { de color* }\end{array}$ & $\begin{array}{l}\text { Cambio } \\
\text { de brillo* }\end{array}$ & Desgaste* & $\begin{array}{l}\text { Manchado } \\
\text { de clavos* }\end{array}$ & Grietas* & $\begin{array}{l}\text { Presencia } \\
\text { de mohos* }\end{array}$ \\
\hline 1 & $2,80 \mathrm{e}$ & $2,80 \mathrm{e}$ & $2,89 \mathrm{~g}$ & $2,40 \mathrm{e}$ & $3,92 \mathrm{f}$ & $3,50 \mathrm{~b}$ \\
\hline 2 & $3,23 \mathrm{f}$ & $3,23 \mathrm{f}$ & $3,23 \mathrm{~h}$ & $1,52 \mathrm{~d}$ & $4,00 \mathrm{f}$ & $3,70 \mathrm{~d}$ \\
\hline 3 & $0,65 a$ & $0,70 \mathrm{a}$ & $0,67 a b$ & $0,47 a b$ & $0,84 \mathrm{~b}$ & $3,80 \mathrm{de}$ \\
\hline 4 & $0,80 a$ & $0,84 \mathrm{a}$ & $0,83 \mathrm{~b}$ & $0,49 \mathrm{~b}$ & $1,14 b$ & $3,80 \mathrm{de}$ \\
\hline 5 & $0,52 \mathrm{a}$ & $0,60 \mathrm{a}$ & $0,49 a$ & $0,57 \mathrm{~b}$ & $0,90 \mathrm{~b}$ & $3,90 \mathrm{de}$ \\
\hline 6 & $1,45 \mathrm{~b}$ & $1,44 \mathrm{~b}$ & $1,50 \mathrm{~cd}$ & $1,33 \mathrm{~cd}$ & $2,11 \mathrm{c}$ & $4,00 \mathrm{e}$ \\
\hline 7 & $1,45 b$ & $1,42 b$ & $1,51 \mathrm{~cd}$ & $1,10 \mathrm{c}$ & $2,40 \mathrm{c}$ & $4,00 \mathrm{e}$ \\
\hline 8 & $1,80 \mathrm{c}$ & $1,80 \mathrm{c}$ & 1,84 e & $2,39 \mathrm{e}$ & $2,28 \mathrm{c}$ & $3,92 \mathrm{de}$ \\
\hline 9 & $1,87 \mathrm{c}$ & $1,82 \mathrm{c}$ & $1,66 \mathrm{de}$ & $3,13 \mathrm{f}$ & $1,02 \mathrm{~b}$ & $3,00 \mathrm{c}$ \\
\hline 10 & $2,31 \mathrm{~d}$ & $2,28 d$ & $2,37 \mathrm{f}$ & $1,40 \mathrm{~d}$ & $3,33 \mathrm{e}$ & $2,40 \mathrm{a}$ \\
\hline 11 & $1,32 b$ & $1,32 b$ & $1,30 \mathrm{c}$ & $1,53 \mathrm{~d}$ & $2,87 \mathrm{~d}$ & 4,00 e \\
\hline 12 & $0,61 \mathrm{a}$ & $0,61 \mathrm{a}$ & $0,61 \mathrm{ab}$ & $0,20 a$ & $0,42 \mathrm{a}$ & $4,00 \mathrm{e}$ \\
\hline $\mathrm{p}^{\star *}$ & 0,0000 & 0,0000 & 0,0000 & 0,0000 & 0,0000 & 0,0000 \\
\hline
\end{tabular}

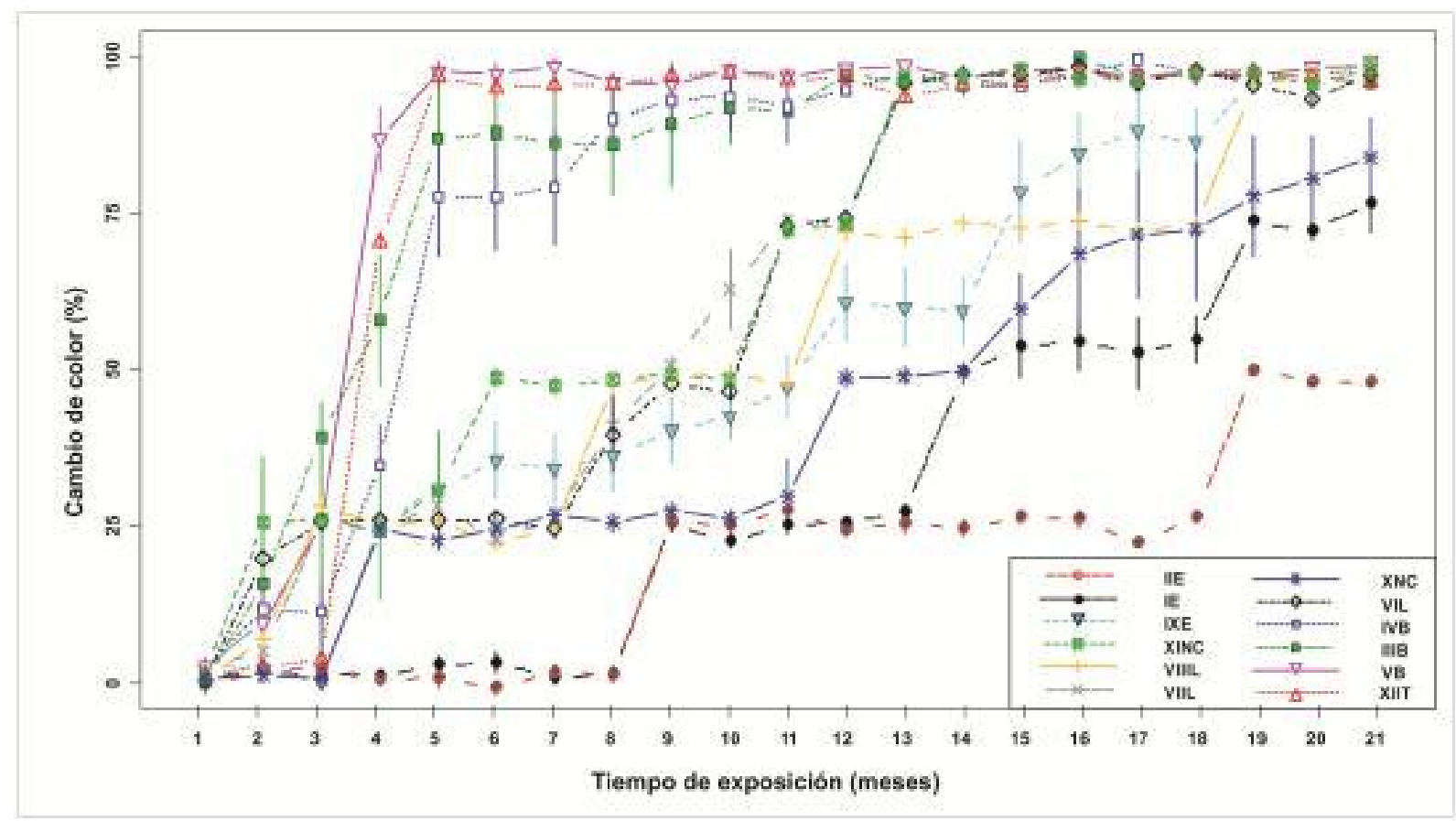

Figura 1. Evolución de cambio de color con el tiempo de exposición.

Asimismo en términos generales a partir de los 9 meses de exposición se visualizó una fuerte degradación cromática del material tomando un color grisáceo característico del intemperismo.

\section{Manchado de Clavos}

De la Tabla 1 se desprende que el esmalte acrílico (9) fue el que presentó el mejor comportamiento mientras que el testigo (12) y los barnices $(3,4$ y 5 ) perdieron la vida útil rápidamente. En particular el esmalte acrílico (9) utilizado en este ensayo fue el único que se diferenció del resto (Figura 2). Este mismo presentó menor proporción de manchas de clavos con respecto al resto de los tratamientos, comportamiento posiblemente asociado a la lixiviación de dichos tintes. 


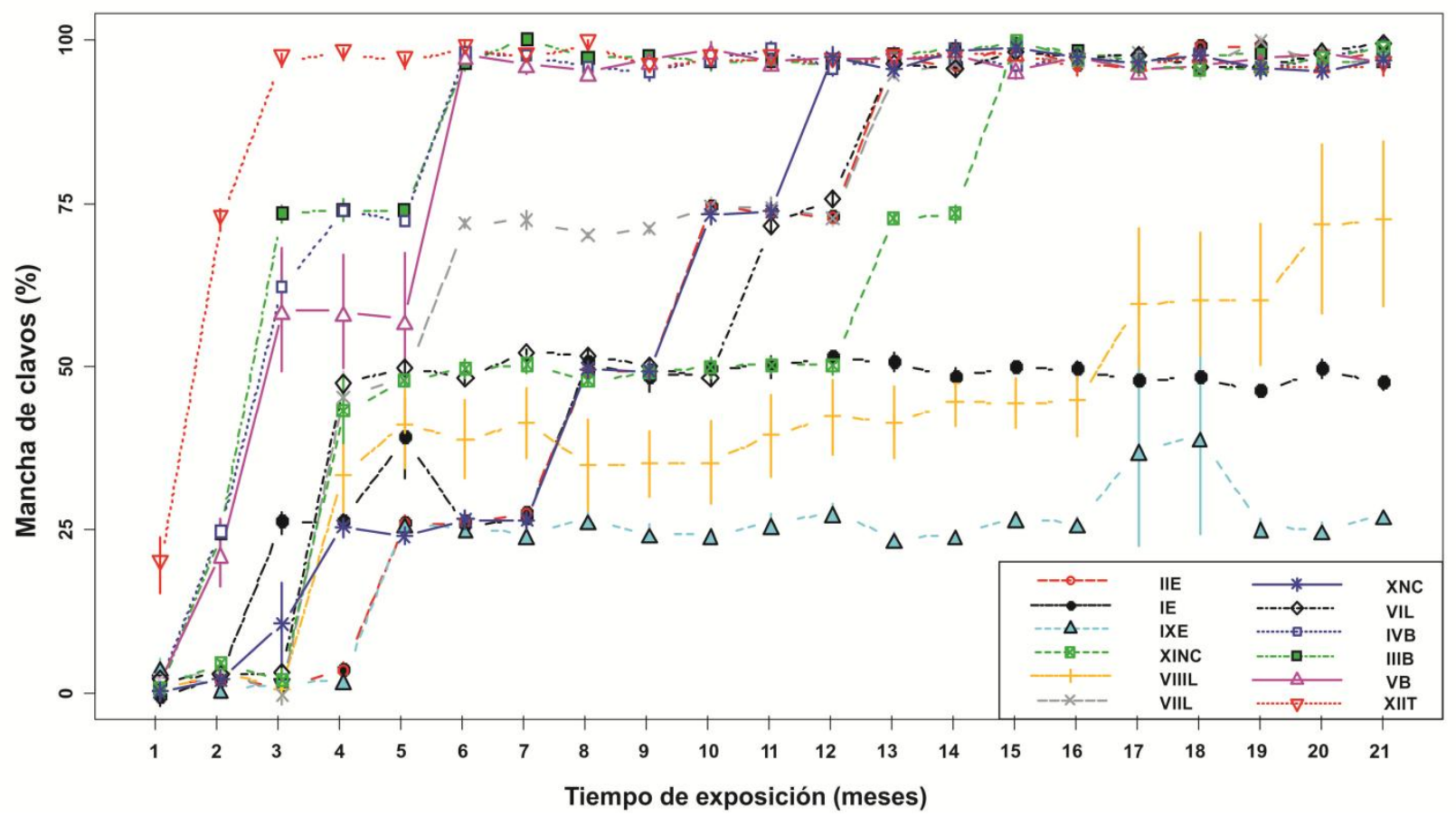

Figura 2. Evolución de manchas de clavo con el tiempo de exposición.

\section{Presencia de grietas}

En cuanto a la formación de grietas los recubrientes que mejor se comportaron fueron el esmalte sintético al agua (2) y el esmalte sintético al solvente (1), estos aíslan a la madera de los cambios dimensionales y por tanto la hacen menos propensa a la formación de grietas. Según el Test de Tukey (Tabla 1) ambos tratamientos se diferenciaron del resto, aunque únicamente el esmalte al agua evitó la formación de grietas durante los 21 meses de exposición (Figura 3).

\section{Presencia de mohos}

Contrariamente a lo sucedido con las otras variables analizadas, los esmaltes $(1,2$ y 9$)$ y el lasur semitransparente (10) fueron los que mayor cantidad de mohos en la superficie mostraron (Figura 4), este comportamiento podría estar asociado a la formulación de los productos, con mayor proporción de fungicidas en los productos de mejor comportamiento (Tabla 1). A su vez el testigo (12) se mantuvo libre de mohos durante la mayor parte del ensayo, comportamiento probablemente vinculado al bajo contenido de humedad de la superficie, debido al libre intercambio de humedad con la atmósfera por no presentar recubrimiento, con lo que tampoco se evidenció crecimiento de microorganismos.

\section{Índice de vida útil}

En la Figura 5 se observa el decaimiento, expresado como índice de vida útil, de los 12 tratamientos evaluados durante el tiempo de exposición (21 meses).

De acuerdo con la Figura 5, se puede diferenciar el decaimiento de recubrientes (tratamientos superficiales) en 3 grupos. El primer grupo constituido por esmalte sintético al agua (2) y al solvente (1), los cuales no evidenciaron la pérdida de vida útil durante el ensayo; un segundo grupo constituido por lasures $(6,7,8,10$, 11) y esmalte acrílico (9) que evidenciaron un progresivo decaimiento a través del tiempo; por último, un tercer grupo constituido por barnices $(3,4,5)$ y testigo (12)que evidenciaron un rápido decaimiento a los 3 meses de exposición con una consecuente pérdida temprana de vida útil.

\section{DISCUSIÓN}

Transcurrido el primer mes de exposición se observó el decaimiento de los recubrientes, especialmente en el cambio de color, comportamiento similar a lo mencionado por Williams (2005). Rivera Nava et al., (2016) estudiando el intemperismo acelerado en maderas de Cedrela odorata L. y Roseodendron donnell-smithii (Rose) Miranda reportaron una vida útil de barnices de 1 a 9 años dependiendo la pintura utilizada y de las propiedades físicas de la madera. Las especies mencionadas poseen un valor de anisotropía por debajo de 2, maderas estables, en comparación con el material utilizado en este trabajo que presenta valores altos de coeficiente de anisotropía de 2,4\%; por lo que es un material propenso a la formación de alabeos, grietas y/o rajaduras (Keil et al., 2017). El comportamiento de la madera indica que a mayor coeficiente de anisotropía menos estabilidad dimensional y por tanto mayor probabilidad de generar grietas y/o rajaduras. 


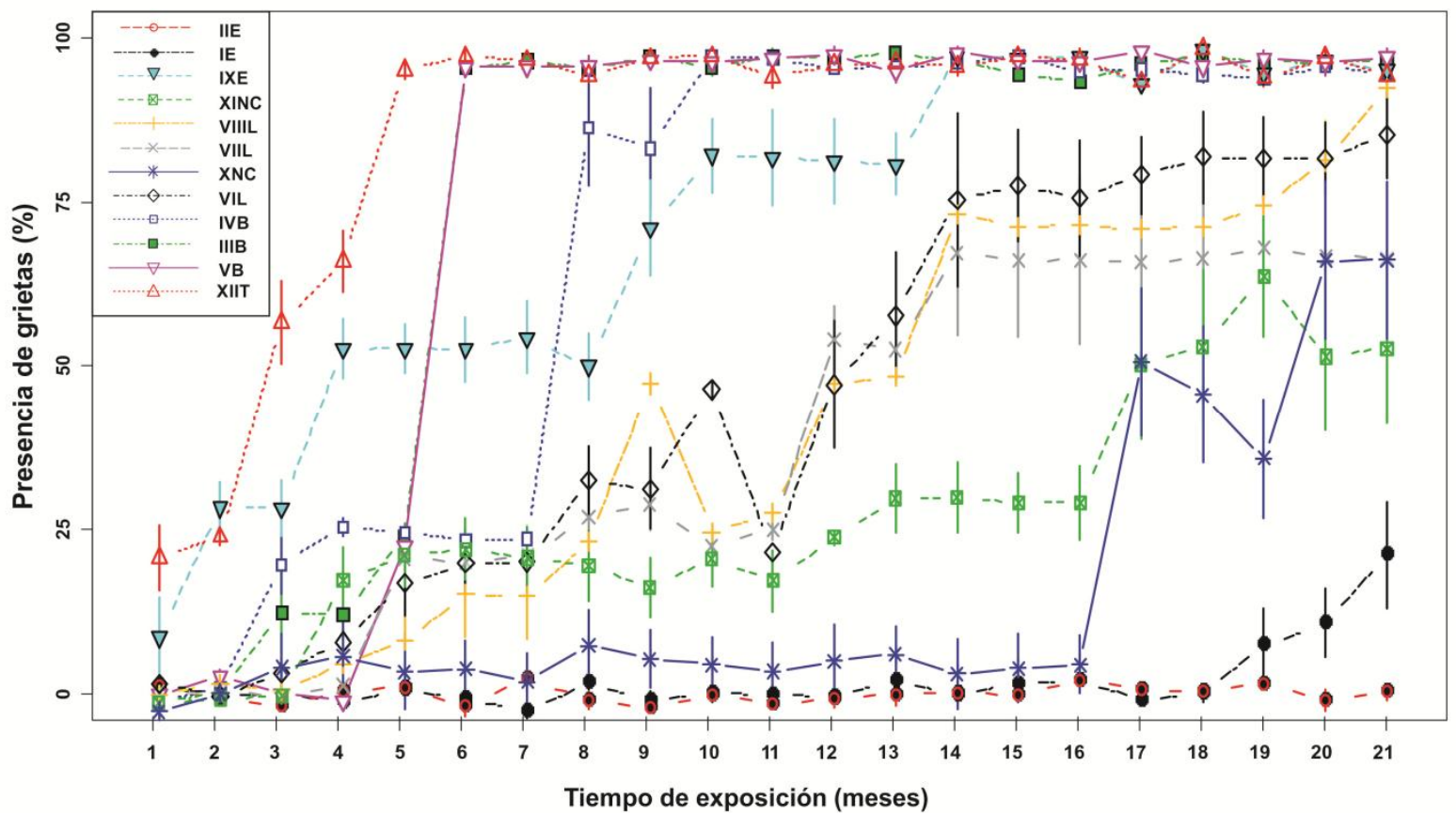

Figura 3. Evolución de la aparición de grietas con el tiempo de exposición.

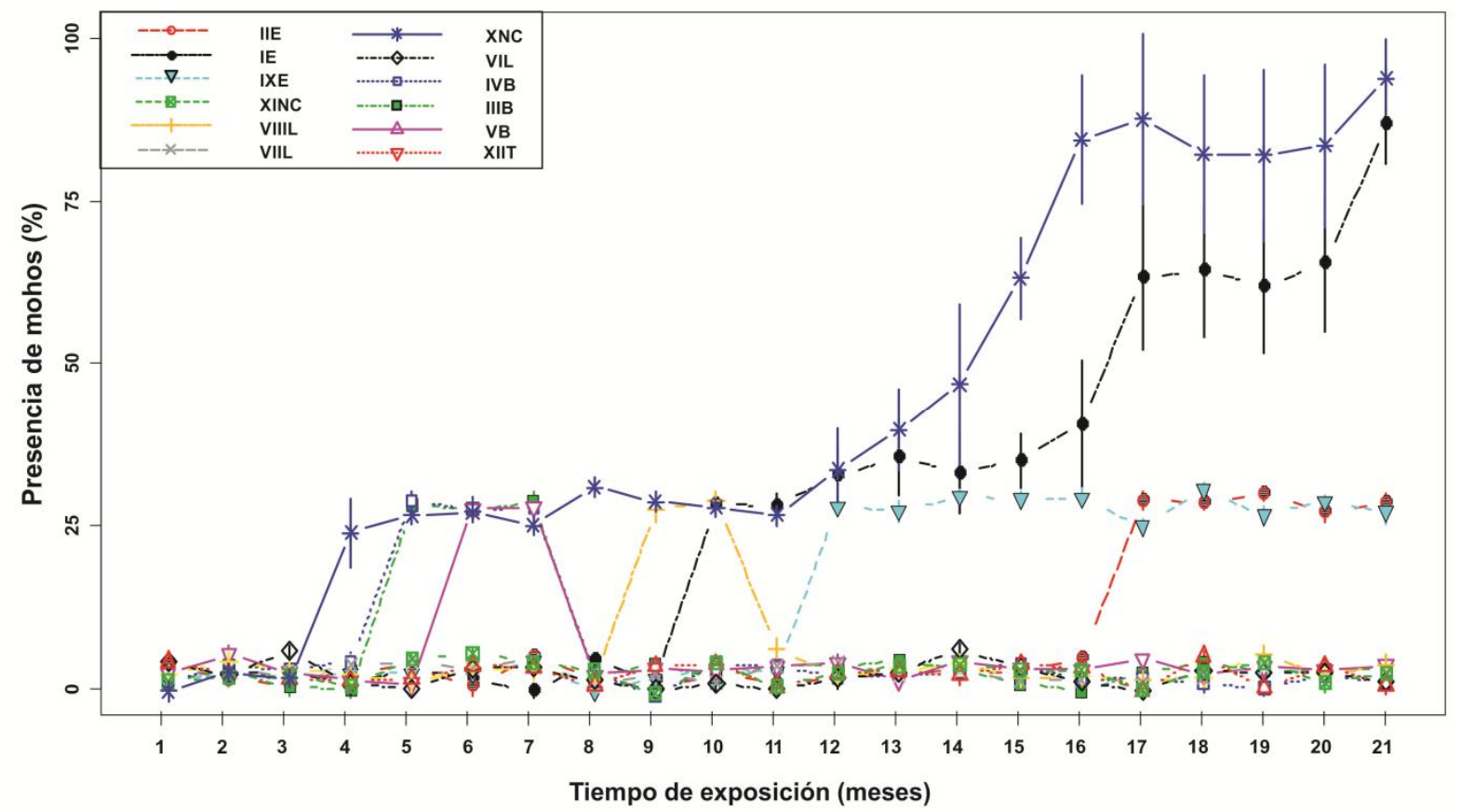

Figura 4. Evolución de la aparición de mohos con el tiempo de exposición.

Coincidentemente con lo hallado en este estudio, Garay Moena (2007) evaluando la vida útil de acabados tipo lasur en tableros OSB y contrachapados, determinó que los lasures no satisfacen completamente la protección necesaria bajo condiciones severas, asimismo producen cambios en las propiedades mecánicas y físicas de la madera siendo los acabados que permiten el intercambio de gases con la atmósfera. 


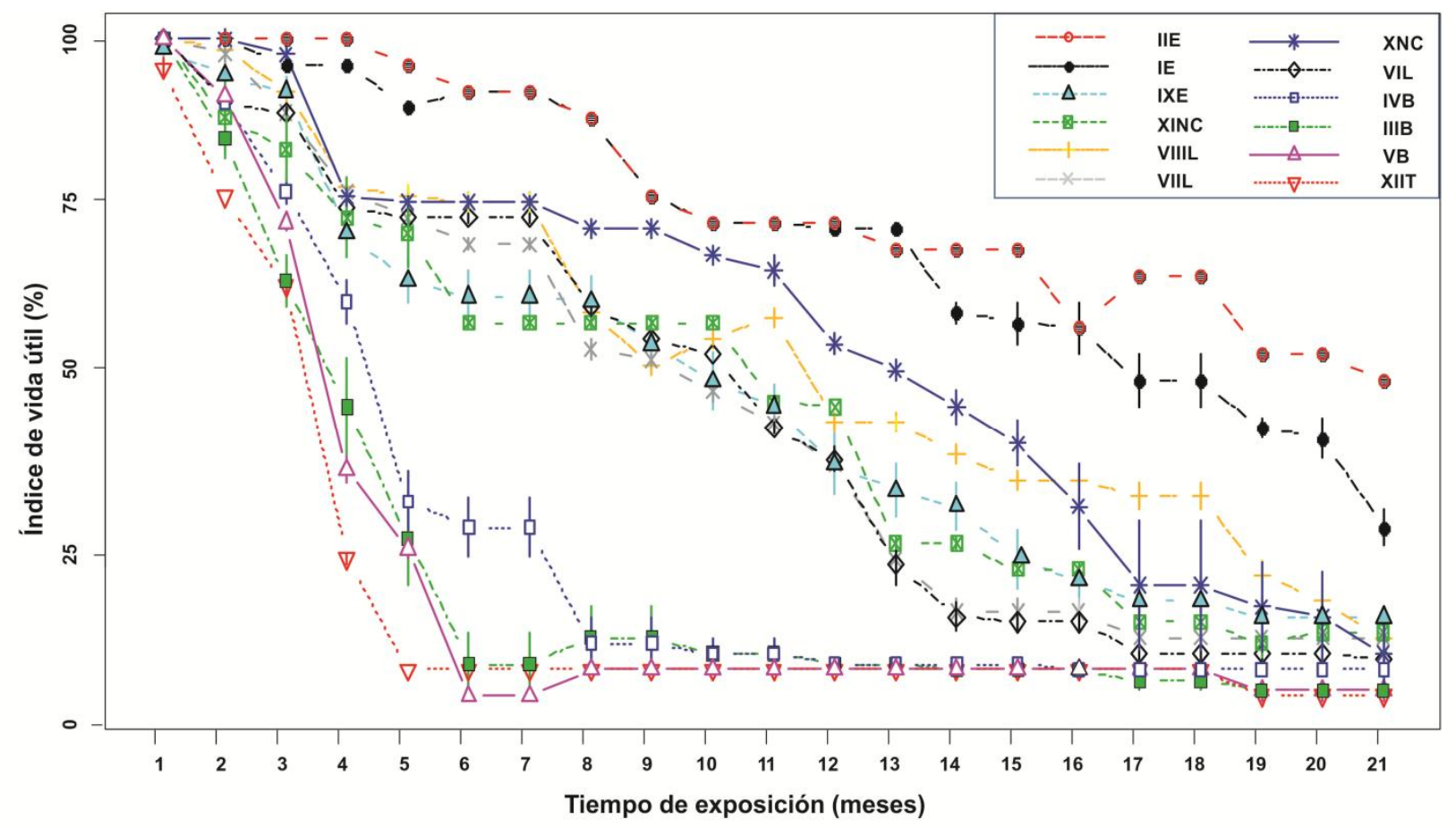

Figura 5. Indice de vida útil de los recubrientes en el período considerado.

\section{Cambio de Color, brillo y desgaste}

Según Williams (2009), el recubriente pierde la vida útil cuando sufre una pérdida de brillo o desgaste; dadas las condiciones meteorológicas del ensayo, el mayor desgaste ocurrió durante un fuerte período de lluvias en el tercer mes de exposición (Boletín agro-meteorológico EEJH, 2013) donde, si bien la radiación solar fue baja, las lluvias pudieron potenciar la lixiviación de los componentes degradados por los UV, y el aumento del contenido de humedad en la madera pudo provocar hinchamientos y contracciones posteriores que favorecieron la aparición de grietas y/o rajaduras, desgastando y agrisando el acabado superficial.

El cambio de color y pérdida de brillo a ojo desnudo se comenzó a percibir a partir los 60-100 días de exposición, dependiendo el recubriente, coincidiendo, en términos generales, con lo encontrado por Valverde \& Moya (2010) y González de Cademartori et al. (2015) en la mayoría de los recubrientes. En concordancia con los resultados de este trabajo, Hunt et al. (2003) demostraron que los acabados de color blanco se comportan mejor, en cuanto al agrietamiento del sustrato y del recubriente, que los que contienen pigmentos de otro color. La degradación cromática de los recubrientes puede estar provocada fundamentalmente por los rayos UV (Avila Delucis et al., 2016).

\section{Manchado de Clavos}

El ensayo experimentó períodos de precipitaciones que aumentaron significativamente el contenido de humedad $(\mathrm{CH})$. Este aumento pudo provocar, entre otros fenómenos, el desprendimiento de las sales de hierro otorgándole a la madera una coloración azul oscuro característico (Carranza et al., 2010). Asimismo la combinación de hierro y madera ocasiona una disminución de la vida útil en las estructuras, fundamentalmente si se encuentran en ambientes agresivos como cercanía al mar o sectores industriales, producto dela aceleración del proceso de corrosión de los elementos metálicos de acero (Borallo Jiménez, 2007).

\section{Presencia de grietas}

Los cambios dimensionales provocados por las diferencias en el $\mathrm{CH}$ de la madera originaron grietas y/o rajaduras posiblemente debidas, entre otras cosas, a la degradación de la laminilla media tal como lo expresa la bibliografía (Acevedo et al., 2013; Williams, 2005; Flores-Velázquez et al., 2001; Cassens \& Feist, 1991). En términos generales se visualizó durante los 21 meses de exposición a ojo desnudo que las grietas se formaron en la transición entre madera temprana y madera tardía, tal como reporta Williams (2009). A su vez, coincidentemente con lo señalado por Williams (2005) y González de Cademartori et al. (2015), la presencia de nudos y medula acentuaron la formación de las mismas provocando un mayor porcentaje de anomalías en la madera intemperizada. En cuanto a los recubrientes utilizados, en algunos casos la falla se evidenció tempranamente debido, posiblemente, a la rigidez de los mismos que no acompañaron los movimientos de la madera, como en los casos del esmalte acrílico (9) y los barnices (3, 4 y 5) (FloresVelázquez et al., 2001). Las pinturas tipo lasur al ser microporosas revelan un comportamiento que acompañan los movimientos de la madera. Hunt et al. 
(2003) observaron que las pinturas látex ofrecieron una menor probabilidad de agrietamiento de la superficie de la pintura y del sustrato.

\section{Presencia de mohos}

El desarrollo fúngico tuvo lugar en la etapa final del ensayo de intemperismo, en este caso a partir del decimocuarto mes afectando posiblemente las propiedades físico-químicas del material. Coincidentemente con los estudios de algunos autores, se encontró una menor colonización fúngica en los recubrientes semitransparentes, pudiendo estar asociado a un menor contenido de humedad debido intercambio gaseoso dado por este tipo de recubrientes. La colonización de mohos en la madera condiciona la madera a colonización por otros agentes biológicos, tales como hongos de pudrición (Avila Delucis et al., 2016; Gobakken \& Westin, 2008; Morrel, 2008; Garay Moena, 2007).

\section{CONCLUSIONES}

En base a los resultados cualitativos obtenidos de este trabajo queda en evidencia que no existe un tipo de recubriente que atienda a todos los factores abióticos y que no sufra la acción del intemperismo. A su vez, el material empleado presenta propiedades específicas, intrínsecas de esta especie, que no se puede aislar y generalmente empeoraron la vida útil del recubriente, como fue el caso de las anomalías y alta anisotropía. Es por ello, que resulta necesario conocer su uso y la condición de intemperie a la que se expondrá el material para luego decidir qué tipo de recubriente utilizar.

\section{Agradecimientos}

A CORFONE (Corporación Forestal Neuquina) por brindar los espacios y el material de ensayo. Al Dr. Manuel Cellini por el aporte de opiniones que mejoraron el trabajo.

\section{BIBLIOGRAFÍA}

Acevedo, A., C. Bustos, J.P. Lasserre \& W. Gacitua. 2013. Efecto de un envejecimiento acelerado mediante rayos UV en la propagación superficial de grietas de debobinado en tableros contrachapados de Eucalyptus nitens. Maderas. Ciencia y tecnología 15(1): 45-56.

Anderson, E., Z. Pawlak, N. Owen \& W. Feist. 1991. Infrared Studies of Wood Weathering. Part I: Softwoods. Society for Applied Spectroscopy 45(4): 7 pp.

Avila Delucis, R., R. Herrera Diaz, J. Labidi \& D.A. Gatto. 2016. Color de la madera de dos eucaliptos expuestas al intemperismo natural en tres entornos ambientales diferentes. Maderas. Ciencia y tecnología 18(1): 133-142. DOI: 10.4067/S0718221X2016005000014.

Boletín Agrometeorológico 2013. Climatología y Fenología Agrícola y Estación Experimental "Ing. Agr. Julio Hirschhorn", Facultad de Ciencias Agrarias y Forestales, Universidad Nacional de La Plata.
Borallo Jiménez, M. 2007. Construcción en madera: influencia de los protectores de madera basados en sales hidrosolubles en la corrosión de herrajes metálicos. Revista Informes de la Construcción 59 (506): 43-49.

Carranza, R.M., G. Duffo \& S. Farina. 2010. Nada es para siempre, química de la degradación de los materiales. Colección: las ciencias naturales y la matemática. Ministerio de Educación. Instituto Nacional de Educación Tecnológica. 155 pp.

Cassens, D. \& W. Feist. 1991. Exterior Wood in the South: Selection, Aplications and finishes. Gen. Tech. Rep. FPL-GTR-69. Madison, WI. United States Department of Agriculture. Forest Service, Forest Products Laboratory. 60 pp.

Chang, ST., D. Hon \& W. Feist. 1982. Photodegradation and photoprotection of wood. Wood and Fiber 14(2): 104-117.

Evans, P., M.J. Chowdhury, B. Mathews, K. Schmalzl, S. Ayer, M. Kiguchi \& Y. Kataoka. 2005. Weathering and Surface Protection of Wood. Handbook of Environmental Degradation of Materials, Chapter 14. 21 pp. DOI:10.1016/B978-081551500-5.50016-1.

Flores-Velázquez, R., A. Borja de la Rosa, F. Zamudio-Sánchez, M. Fuentes-Salinas \& E. González-Estrada. 2001. Determinación a través de pruebas aceleradas, de la vida útil del acabado para exteriores en madera de pino y encino. Revista Chapingo Serie Ciencias Forestales y del Ambiente 7(1): 99-105.

Gascón-Garrido, P., N. Mainusch, H. Militz, W. Viöl \& C. Mai. 2016. Effects of copper-plasma deposition on weathering properties of wood surfaces. Applied Surface Science 366: 112-119. DOI: 10.1016/j.apsusc.2016.01.060.

Garay Moena. R.M. 2007. Impregnantes tipo lasur para la protección superficial de maderas y tableros. Revista Agro-Ciencia 23(1): 25-36.

Gobakken, L.R. \& M. Westin. 2008. Surface mould growth on five modified wood substrates coated with three different coating systems when exposed outdoors. International Biodeterioration \& Biodegradation 62: 397402.

González de Cademartori P.H., A.L. Missio, B. Dufau Mattos \& D.A. Gatto. 2015. Natural Weathering Performance of Three Fast-Growing Eucalypt Woods. Maderas. Ciencia y tecnología 17(4): 799 - 808.

Hunt, M.O., A.J. O'Malley, W.C. Feist, G.P. McCabe, J.W. Evans II \& D.L. Cassens. 2003. Weathering of painted wood construction: Facade restoration. Forest Products Journal 53(4): 51-60.

Keil, G., E. Spavento, M. Murace, M. Tonello, M. Luna, G. Acciaresi, L. Maly, M. Refort \& I. Andía. 2017. Mejoras tecnológicas en la madera juvenil de pino ponderosa (Pinus ponderosa Douglas ex Laws) para usos en productos sólidos y/o encolados. Proyecto de Investigación Aplicada (PIA 10011). Publicación del 2017. pp. 343-346.

Keil, G., C. Taraborelli, M. Refort, L Maly \& R. Cámera. 2016. Evaluación de recubrimientos de uso exterior aplicados en madera de Pino ponderosa (Pinus ponderosa Douglas exLaws) expuesta en el partido de La Plata, Argentina. V Congreso Iberoamericano de Protección de la Madera RIPMA. 10 pp. 
Keil, G, E. Spavento, E. Muñoz, S. Alegre, C. Taraborelli \& M. Refort. 2015. Construcción en madera: acción conjunta entre organismos estatales de educación/ extensión e investigación, una experiencia Argentina. Revista Ciência da Madeira (Brazilian Journal of Wood Science) 6(3): 254-263. DOI: 10.12953/2177-6830/rcm.v6n3p254-263

Morrel, J.J. 2008. Molds and Stain Fungi. Downloaded by North Carolina State Univ. Disponible en: http://pubs.acs.org. Ultimo acceso: August 4, 2012.

R Core Team. 2018. R: A language and environment for statistical computing. R Foundation for Statistical Computing, Vienna, Austria. URL https://www.Rproject.org/.

Rivera Nava, J.L., A. Borja de la Rosa, R. FloresVelázquez \& A. Corona Ambriz. 2016. Evaluación de dos barnices mediante intemperismo acelerado, aplicados en madera de plantaciones. Madera y bosques 22(3):

112. https://dx.doi.org/10.21829/myb.2016.2231459.

Rodríguez-Anda R. \& F.J. Fuentes-Talavera. 2003. Factores que intervienen en el proceso de envejecimiento de la madera. Revista Chapingo Serie Ciencias Forestales y del Ambiente 9(1): 95-100.

Tamarit-Urias J.C., A. Borja-de la Rosa, R. FloresVelázquez \& A. Corona-Ambríz. 2003. Vida útil de dos barnices para exteriores mediante intemperismo artificial en madera de aile, pino y encino. Revista
Chapingo Serie Ciencias Forestales y del Ambiente 8(1): 83-90.

Taraborelli C. 2016. Evaluación de recubrimientos de uso exterior aplicados en madera de Pino ponderosa (Pinus ponderosa Douglas ex. Laws). Tesis de grado (Ingenieríaa Forestal). Facultad de Ciencias Agrarias y Forestales, Universidad Nacional de La Plata, La Plata, Argentina. $43 \mathrm{pp}$

UNE-EN 927-3. 2000. Pinturas y barnices: materiales de recubrimiento y sistemas de recubrimiento para madera exterior. Ensayo de envejecimiento natural, Parte 3.

Valverde, J.C. \& R. Moya. 2010. Efectos de la intemperie en el color de dos acabados aplicados en madera de Cedrela odorata y Carapa guianensi. Maderas. Ciencia y Tecnología 12(3): 171-180.

Williams, S.R. 2005. Weathering of Wood. Handbook of Wood Chemistry and Wood Composites. Chapter 7. USDA Forest Service, Forest Products Laboratory, Madison, WI. pp. 139-185.

Williams, S.R. 2009. Testing Painted Wood: Past Practices at the Forest Products Laboratory and Recommendations for Future Research. Third International Coating Wood and Wood Composites Conference: durable and sustainable--today and beyond, Charlotte, NC. National Paint \& Coatings Association, Washington, D.C. 